\title{
The Management of Paediatric Crohn's Disease: Addressing Unmet Needs
}

\author{
Geneviève Veereman-Wauters ${ }^{*}, 1$ and Salvatore Cucchiara ${ }^{2}$ \\ ${ }^{I}$ Division of Pediatric Gastroenterology, Hepatology and Nutrition, Queen Paola Children's Hospital ZNA \& University \\ Hospital Antwerp, Belgium \\ ${ }^{2}$ Sapienza University of Rome, Pediatric Gastroenterology \& Liver Unit, Center of Pediatric IBD, Italy
}

\begin{abstract}
Paediatric Crohn's disease (CD) affects 5 in 100,000 children in the US and Europe and can result in growth retardation and delayed sexual development. Therefore, early diagnosis and treatment is critical, with the goal being maintenance of symptomatic remission and a change in disease course. Conventional treatment relies on aminosalicylate maintenance therapy with corticosteroids to control acute exacerbations and immunomodulators for steroid-resistant or frequently relapsing disease. Infliximab has demonstrated efficacy in moderately to severely active paediatric $\mathrm{CD}$, with $88.4 \%$ patients in clinical response and $58.9 \%$ in clinical remission at week 10 . Significant improvements in quality of life, height, reduction in corticosteroid use and mucosal healing were observed with infliximab. Traditional 'step-up' treatment strategy may be suboptimal because relapse and steroid dependency/resistance rates remain high. A 'top down' approach using biologic therapy earlier may suppress intestinal inflammation and promote prolonged and stable remission, but safety issues need to be considered.
\end{abstract}

\section{INTRODUCTION}

Although adult and paediatric Crohn's disease (CD) are similar, there are a number of clinical features unique to children [1]. In addition to the gastrointestinal (GI) symptoms (e.g., abdominal pain, diarrhoea, weight loss, loss of appetite, etc), children and adolescents often experience a range of extraintestinal manifestations including arthritis as well as delayed growth and puberty (Table 1) [1]. Up to $35 \%$ of paediatric patients with inflammatory bowel disease (IBD) will present with at least one extraintestinal manifestation $[2,3]$, the most common of which is arthritis, which affects up to $25 \%$ of children [4] and often manifests before the GI symptoms are detected [5]. Skin manifestations are also common, including erythema nodosum and pyoderma gangrenosum $[1,6]$.

$\mathrm{CD}$ has a tremendous effect on the general health and nutritional status of paediatric patients [1]. Increased metabolic demands and decreased intake lead to nutritional deficiencies $[1,6]$. This is further exacerbated by disease-related weight loss/anorexia, decreased appetite and decreased absorptive capacity [6]. The effect of nutritional insufficiency has a greater impact on children than adults because of the increased nutritional requirement needed to maintain normal rapid periods of growth during childhood and adolescence $[1,6]$. Poor nutritional status [7] as well as intestinal losses, protein-losing enteropathy and inflammation can lead to growth retardation and delayed sexual development [8]. At diagnosis, up to $85 \%$ of children with $\mathrm{CD}$ have growth failure [9]. The earliest and most common sign is reduced linear growth, with $46 \%$ of children exhibiting abnormalities

\footnotetext{
*Address correspondence to this author at the Division of Pediatric Gastroenterology, Hepatology and Nutrition, Queen Paola Children's Hospital ZNA \& University Hospital Antwerp, Belgium; Tel: +3232802101; Fax: +3232802133; E-mail: gigi.veereman@zna.be
}

Table 1. Extraintestinal Manifestations Observed in Paediatric Patients with CD

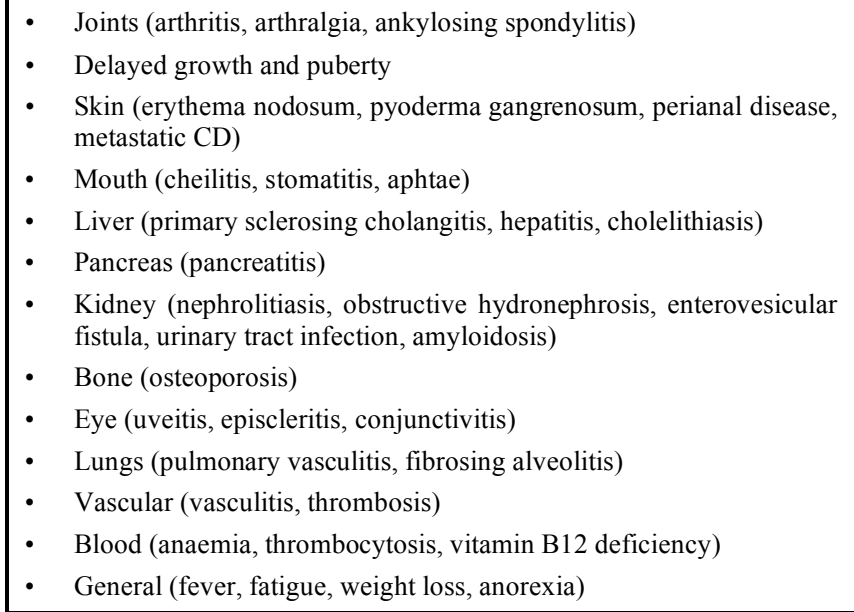

Reference [1]. Adapted with permission from Elsevier Limited.

before the onset of GI symptoms [10]. Furthermore, weight loss is observed in about $85 \%$ of children with $\mathrm{CD}$ [9]. Puberty is commonly delayed, with active disease slowing or even stopping the progression of sexual development [1]. Effective treatment is very important because maintaining disease remission prior to puberty usually results in improved and catch-up growth [8]. In addition to the physical consequences of $\mathrm{CD}$, there are also psychosocial complications. Many children are concerned about their energy levels and body image. They fail to be in control of their daily activities; furthermore, delayed puberty has a detrimental effect on self-esteem and social interaction [1]. It is therefore clearly important to diagnose and treat paediatric CD early in order to not only induce and maintain remission, but also to address nutritional deficiencies, ensure normal growth and 
development, improve quality of life (QoL) and change the course of disease.

\section{AN INCREASING PROBLEM}

The incidence of $\mathrm{CD}$ has been increasing in children of all ages [6]. Although the incidence of CD is lower in children than in adults, there has been a rapid rise over the last few decades, which now seems to be stabilising [11-13]. In the UK, the incidence of CD rose from 1.3 per 100,000 between 1983 and 1988 to 3.11 per 100,000 between 1989 and 1993, a threefold increase over 10 years [14]. More recent epidemiological data indicates that the incidence of $\mathrm{CD}$ is now approximately 5.0 per 100,000 in the North American and European paediatric populations, with $25 \%$ to $30 \%$ of all CD patients being diagnosed before age 20 years $[15,16]$. A slightly higher incidence of 5.2 per 100,000 children per year has been observed in children younger than 16 years with IBD in the UK and the Republic of Ireland [17].

\section{DIAGNOSING PAEDIATRIC CD}

The symptoms of CD in children can be difficult to diagnose, depend on disease location and extent of inflammation and vary from person to person [1]. The most common GI symptoms are abdominal pain and diarrhoea. Other GI symptoms include anorexia, weight loss, nausea, vomiting, rectal bleeding and perianal disease [18]. IBD can also affect the mental health of patients: adolescents with IBD have a higher risk for psychiatric disorders [19]. Growth retardation and delayed puberty may be the only presenting signs [18]. These result in insufficient nutrition, malabsorption, protein losing enteropathy and inflammation [8].

Diagnosis of CD requires a thorough patient and family history and physical examination [20], with particular attention paid to growth and pubertal stage [6]. Physical examination will reveal the presence of extraintestinal manifestations [18]. Abdominal examination tends to be non-specific, but the presence of a mass in the right lower quadrant may point to $\mathrm{CD}$. It is imperative to carry out a rectal examination to identify faecal blood or perianal disease [6]. Laboratory tests are not specific [6] but indicate inflammation, organ involvement (e.g., liver) and nutritional deficiencies [21]. Serological markers may be helpful in differentiating the type of IBD [22]. The Pediatric Crohn's Disease Activity Index (PCDAI) is a validated score based on history, clinical examination and simple laboratory tests (complete blood count $[\mathrm{CBC}]$, erythrocyte sedimentation rate [ESR], albumin) to evaluate the severity of disease [23]. Stool samples are needed for culture to reveal occult bleeding and inflammation (calprotectin) [24]. The gold standard for making a diagnosis of CD is a full endoscopic exploration (upper endoscopy and ileocolonoscopy) with multiple biopsies [20]. In children, these examinations are performed after thorough preparation and under general anesthesia $[25,26]$. In case of small bowel disease, a radiographic study of the small bowel is needed [21]. The video capsule is increasingly being used but obviously has some drawbacks such as the lack of biopsy material and the risk for obstruction [20]. Computed tomography (CT) or magnetic resonance imaging (MRI) scans are needed in case of perforating disease and intra-abdominal abscesses. Full eye examination, bone age and DEXA scan for bone mass are performed once the diagnosis is confirmed. In clinical practice, however, it is often impossible to establish a firm diagnosis of $\mathrm{CD}$ upon the first manifestation of the disease.

\section{PAEDIATRIC CD DISEASE COURSE}

The natural history of paediatric $\mathrm{CD}$ is poorly understood and few population-based studies have been conducted. It has been suggested that in children, there is a high risk of progression to a disabling and complicated disease. Preliminary data from one large, population-based study that identified and followed up (for at least 2 years) all patients with onset of CD before age 17 years in the French 'Registre des Maladies Inflammatories Chroniques de I'Intestin' (EPIMAD) between 1988 and 2002 indicate that childhood-onset $\mathrm{CD}$ is characterised by widespread location at diagnosis (both small bowel and colon, 62\%; colon only, 23\%; small bowel only, $15 \%$ ), a high percentage of patients $(87 \%)$ require corticosteroid therapy with high occurrence of dependence $(24 \%)$ and there is a high probability of colectomy $(8 \%$ after 1 year, $20 \%$ after 3 years, $32 \%$ after 5 years) irrespective of immunomodulator therapy [27].

A pooled analysis involving 1153 children with $\mathrm{CD}$ has also established the disease distribution [28]. It reveals that $38 \%$ of children of all ages have small bowel disease, $38 \%$ have small bowel and large bowel disease and 20\% have large bowel disease. Other studies have shown that upper GI disease occurs in $30 \%$ to $40 \%$ of children [29]. Even higher rates (up to $71 \%$ ) are seen in endoscopic studies [30,31] and perianal disease is found in $11 \%$ to $18 \%[32,33]$. Children under age 5 years have increased incidences of large bowel $(30 \%)$, small bowel and large bowel $(59 \%)$ and perianal disease (34\%) and reduced occurrences of small bowel disease (11\%) [34]. These findings have been confirmed by the ESPGHAN registry [35]. Limited data exist on CD in children under age 1 year and, although $\mathrm{CD}$ is diagnosed in infants, this may represent a particular subgroup of patients [36].

\section{TREATMENT GOALS IN PAEDIATRIC CD}

The therapeutic goals in paediatric CD should go beyond symptomatic remission. The general treatment goals in paediatric $\mathrm{CD}$ are to maintain adequate nutrition status and to optimise the patient's QoL (attending school and taking part in sporting and leisure activities) [6]. In the short term, the main goal should be to induce a rapid response to achieve mucosal healing and maintain symptomatic remission without the use of corticosteroids. In the long term, the management strategy should be to normalise the bowel to prevent relapse; optimise growth and development; avoid complications, hospitalisations, and surgery; as well as prevent disease-related mortality and morbidity. Goals are focused on changing the course of the disease. This means preserving bowel integrity by controlling inflammation and promoting/maintaining complete mucosal healing, including fistulas.

\section{TREATMENT OPTIONS IN PAEDIATRIC CD}

The treatment options available to paediatric patients are similar to those for adults (Table 2). Conventionally, patients with mild disease initially receive an oral aminosalicylate (mesalazine, mesalamine, or sulfasalazine) or antibiotics (e.g., ciprofloxacin or metronidazole). In moderate to severe cases, corticosteroids (prednisone or budesonide) are intro- 
duced [20]. Immunosuppressive agents (azathioprine [AZA], 6-mercaptopurine [6-MP], or methotrexate [MTX]) are prescribed as adjunctive therapy in steroid-resistant patients and to allow steroid tapering in steroid-dependent patients. Based on data from Markowitz [37], immunosuppression tends to be initiated earlier to prevent relapse. Biologic therapies, i.e., infliximab (IFX), are now used in moderate to severe CD (including fistulising disease) refractory to the traditional non-biologic treatments as an adjunct or an alternative to corticosteroids in steroid-resistant patients or in patients not responding to immunosuppressive agents and corticosteroids. IFX is approved for the treatment of severe, active CD in paediatric patients aged 6 to 17 years who have not responded to conventional (non-biologic) therapy including a corticosteroid, an immunomodulator and primary nutrition therapy [38].

Table 2. Treatment Options in Paediatric CD

\begin{tabular}{|c|}
\hline $\begin{array}{ll}\text { - } & \text { Aminosalicylates } \\
\text { - } & \text { Antibiotics } \\
\text { - } & \text { Enteral nutrition } \\
& \text { Corticosteroids } \\
- & \text { Prednisone } \\
- & \text { Budesonide } \\
\text { - Immunomodulators } \\
-\quad \text { 6-mercaptopurine, azathioprine } \\
-\quad \text { Methotrexate } \\
\text { Biologic agents } \\
\text { - Infliximab } \\
\text { Surgery }\end{array}$ \\
\hline
\end{tabular}

Relapse prevention is a key aim of the management of CD. Aminosalicylates are commonly prescribed for the maintenance of remission in $\mathrm{CD}$, despite not being particularly effective. Indeed, a recent meta-analysis of randomised controlled trials (RCT) comparing the effects of aminosalicylates with placebo showed no evidence to suggest that aminosalicylates were of value in maintaining remission in $\mathrm{CD}$ patients [39]. Corticosteroids are the mainstay of treatment for acute exacerbations in children; however, they are ineffective in maintaining remission [40]. In addition, although their chronic use is limited by severe side effects (e.g., moon face/acne, weight gain/redistribution, bone demineralisation, delayed puberty, mood swings, high blood pressure, altered glucose metabolism) [41, 42], patients often receive many rounds of steroid therapy. Tung et al. evaluated the 1-year outcomes of corticoid therapy in paediatric $\mathrm{CD}$ as part of the population-based Olmsted County study [43]. Immediate (30 days) and 1-year outcomes were evaluated. After 30 days, complete remission was seen in $62 \%$ $(n=16)$ of patients and partial remission in $27 \%(n=7)$. After 1 year, $42 \%$ had prolonged response, $31 \%$ were steroid dependent and $27 \%$ required surgery, thus suggesting a need for early use of steroid-sparing therapies in paediatric CD.

Immunomodulators are added in resistant or frequently relapsing patients to maintain remission. In children, 6-MP, when added to corticosteroid therapy, has been shown to significantly reduce the need for prednisone and improve the maintenance of remission [37]. In a study of 55 children (mean age 13) randomised to receive 6-MP or placebo (both groups received prednisone $40 \mathrm{mg} /$ day), only $9 \%$ of $6-\mathrm{MP}-$ treated patients relapsed over an 18-month period compared with $47 \%$ in the placebo group $(P=0.007)$ [37]. Patients treated with 6-MP required fewer days of corticosteroid treatment and were able to remain off of prednisone treatment significantly longer than those receiving placebo. As a preventive measure, the thiopurine methyltransferase (TPMT) genotype of the patient can be assessed prior to initiating AZA or 6-MP in order to lower the risk for bone marrow depression [20].

Enteral nutrition via nasogastric feeding can reverse growth retardation and significantly improve height and weight $[44,45]$. In addition, enteral nutrition can actually induce remission and spare the use of corticosteroids [45], although its use as a primary therapy is controversial. This was highlighted by a meta-analysis comparing the effectiveness of enteral nutrition and corticosteroids [46]. Corticosteroids were shown to be consistently more effective in inducing remission than enteral nutrition [46]. The type of liquid enteral formula (polymeric vs hydrolyses) made no difference. However, in a recent prospective RCT in children with active $\mathrm{CD}$, a polymeric diet for 10 weeks was markedly superior to corticosteroids in promoting healing of inflammatory lesions of the gut [47]. Nutritional supplementation is useful in $\mathrm{CD}$, even during remission; a nutritionist monitors the patient's daily diet to counter deficiencies. Nutritional status can be improved significantly by increasing caloric intake [1].

Surgical intervention is an option in paediatric CD because it may provide a disease-free period that will allow normal growth and development to be restored [48]. Several studies have shown catch-up growth in children following intestinal resection $[49,50]$. In fact, $36 \%$ to $43 \%$ of paediatric patients will require surgery [51,52]. Preliminary data from the EPIMAD registry (population-based incidence cohort diagnosed from 1988-2002) demonstrated that the cumulative risk of surgery in children up to age 17 years with CD was $8 \%, 20 \%$ and $34 \%$ at 1,3 and 5 years, respectively, post diagnosis [53]. This further underlines the need for new treatment options that can induce and maintain remission. Surgery in children is not without risk. Several studies have shown that postoperative clinical recurrence rates are high in children [48]. Furthermore, early clinical recurrence following surgical intervention may further retard growth and development [54]. Baldassano et al. showed clinical recurrence rates in children after surgery to be $17 \%$ after 1 year, $38 \%$ after 3 years and $60 \%$ after 5 years and that recurrence was more likely in patients with severe disease [48].

\section{BIOLOGIC THERAPY IN PAEDIATRIC CD}

Biologic therapies with anti-tumour necrosis factor-alpha (TNF $\alpha$ ) activity, including infliximab (IFX), adalimumab and certolizumab pegol (approved in Switzerland), are available for the treatment of adult CD. The first to be commercially available was IFX, a chimeric, monoclonal antibody that binds both to soluble and transmembrane TNFo and neutralises its activity [55]. IFX was approved for adult CD by the US Food and Drug Administration (FDA) in 1998 and in the EU in 1999. In adults, a single dose of IFX was shown to have a rapid clinical response in the majority of patients $(80 \%)$ with moderate to severe CD resistant to non-biologic 
treatment [56]. In addition, IFX has demonstrated efficacy as maintenance therapy in adult patients with $\mathrm{CD}$ who respond to a single dose of IFX [57]. Adult patients with refractory, moderate to severe CD taking IFX every 8 weeks have been shown to be more likely to maintain prolonged remission, discontinue corticosteroids and achieve mucosal healing [57, 58]. Additionally, in patients with fistulising CD, IFX significantly reduces hospitalisations and surgical procedures [59].

Recent clinical experience has demonstrated that IFX is also effective in paediatric patients. Consequently, in 2006, the US FDA approved IFX for paediatric patients (aged 6 to 17 years) with moderately to severely active CD who have had an inadequate response to conventional therapy [60]. Then, in May 2007, IFX received EU approval for the treatment of severe, active CD in paediatric patients, who have not responded to conventional therapy, including a corticosteroid, an immunomodulator and primary nutrition therapy, or are intolerant to or have contraindications to such therapies [38].

Regulatory approval was granted based on efficacy and safety profile in adult CD patients and on the findings of the phase III REACH trial, a randomised, multicentre, openlabel study that evaluated the safety and efficacy of IFX in paediatric patients with moderate to severe $C D$ [61]. In the REACH trial, 112 patients (aged 6-17 years) with moderate to severely active $C D$ received an induction regimen of IFX $5 \mathrm{mg} / \mathrm{kg}$ at weeks 0,2 and 6 . All patients were required to be taking a concurrent immunomodulator. At week 10, patients who were considered to have clinically responded to induction therapy were randomised to receive IFX $5 \mathrm{mg} / \mathrm{kg}$ every 8 weeks or every 12 weeks as scheduled maintenance therapy. Clinical response was defined as a decrease from baseline in the PCDAI score $\geq 15$ points (total score $\leq 30$ ) and clinical remission as PCDAI score $\leq 10$ points, evaluated every 8 to 12 weeks depending on treatment group up to week 54 .

At week 10, 88.4\% $(\mathrm{n}=99)$ of patients had responded to the induction therapy and $58.9 \%(\mathrm{n}=66)$ were in clinical remission. At week 54, 63.5\% $(n=33)$ and $55.8 \%(n=29)$ of patients receiving IFX every 8 weeks did not require dose

Clinical response at week 54

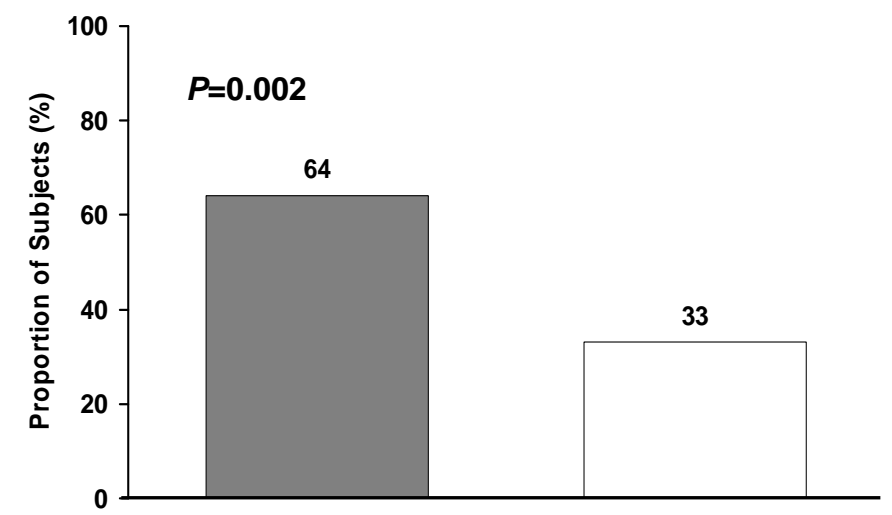

Q8 Week Scheduled adjustment and were in clinical response or clinical remission, respectively, compared to $33.3 \%(n=17)$ and $23.5 \%$ $(n=12)$ of patients, respectively, receiving treatment every 12 weeks (Fig. 1). This demonstrated that paediatric patients responding to IFX induction were more likely to be in clinical response or remission without dose adjustment when scheduled therapy was given every 8 weeks rather than every 12 weeks.

Changes in growth, corticosteroid use and QoL were also evaluated. Average daily corticosteroid use decreased significantly from baseline to week 10 following induction therapy. By the time of the first maintenance dose, $79 \%$ $(19 / 24)$ and $58 \%(7 / 12)$ of patients receiving IFX every 8 weeks and every 12 weeks, respectively, who were on corticosteroids at baseline, had discontinued corticosteroid therapy. The height status of patients with at least a 1-year delay in bone age was also evaluated by calculating the height $\mathrm{z}$ score, a measure of the deviation of the patient's height from that expected for their age/sex. Significant improvements in height were observed at both 30 and 54 weeks (Fig. 2). This is supported by the findings of Walters et al. that showed that IFX improved linear growth (height velocity and height centile) in chronically active, severe paediatric CD patients provided that they were treated before or in early puberty [62].

Furthermore, in the REACH study, QoL was assessed by the IMPACT III questionnaire and it was shown to improve significantly at weeks 10,30 and 54 compared to baseline. No statistically significant differences were seen between the treatment groups. IFX was generally well tolerated, with a safety profile consistent with that seen in adults. Importantly, in the REACH study, there were no incidences of delayed hypersensitivity reaction, death, malignancies, tuberculosis, neurological disorders or autoimmune disorders [61].

In addition to treating the symptoms of CD, IFX has been shown to heal inflammatory lesions in paediatric patients and therefore have an impact on disease progression. Borrelli et $a l$. assessed the effect of IFX on inflammatory remission, defined as a decrease in both endoscopic (Crohn's Disease Endoscopic Index of Severity [CDEIS]) and histological (distal ileum and colon) scores for $\geq 50 \%$ compared to base-

\section{Clinical remission at week 54}

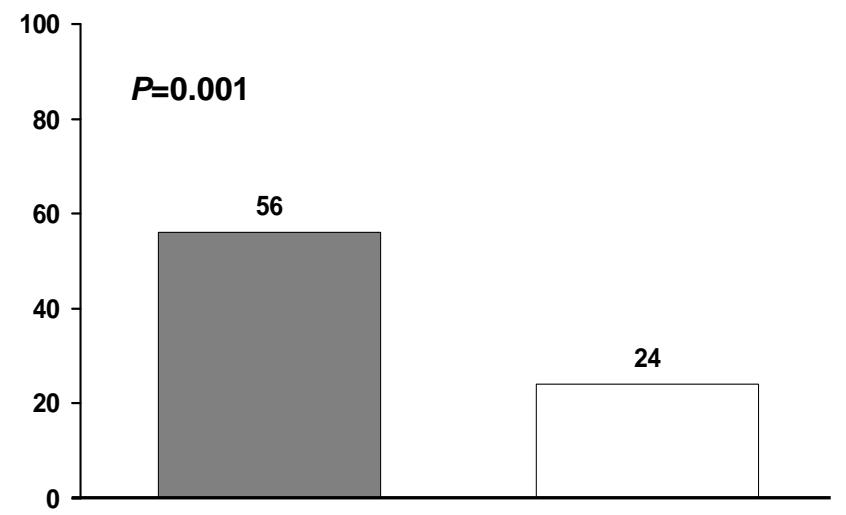

q12 Week Scheduled

Fig. (1). Efficacy of IFX (Q8 vs Q12) in paediatric patients with CD at week 54 [61]. Adapted with permission from Elsevier Limited. 
line [63]. Significant improvements in CDEIS were seen 8 to 10 weeks after IFX induction (Fig. 3), with $66 \%(12 / 18)$ of patients having an inflammatory remission indicating mucosal healing [63].

Randomised patients with $\geq 1$-year delay in bone age $(n=38)$ Median z-score at baseline $=-1.5$

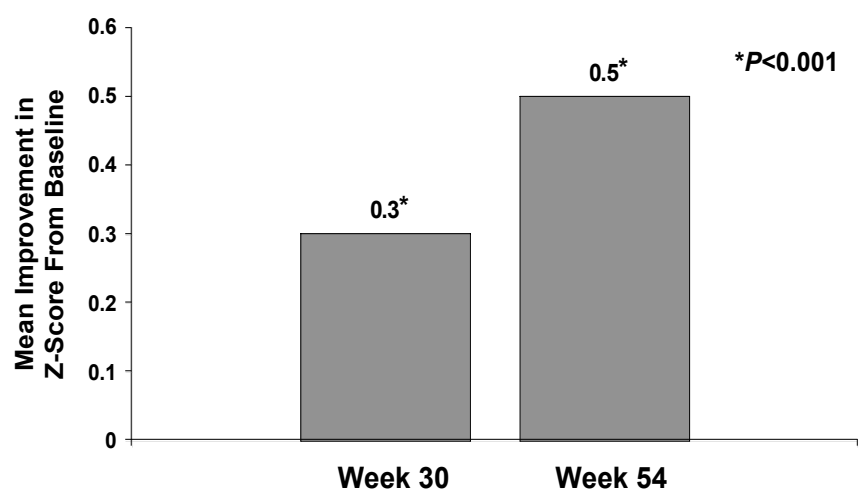

Fig. (2). Significant improvement in height status after 30 and 54 weeks of IFX treatment [61].

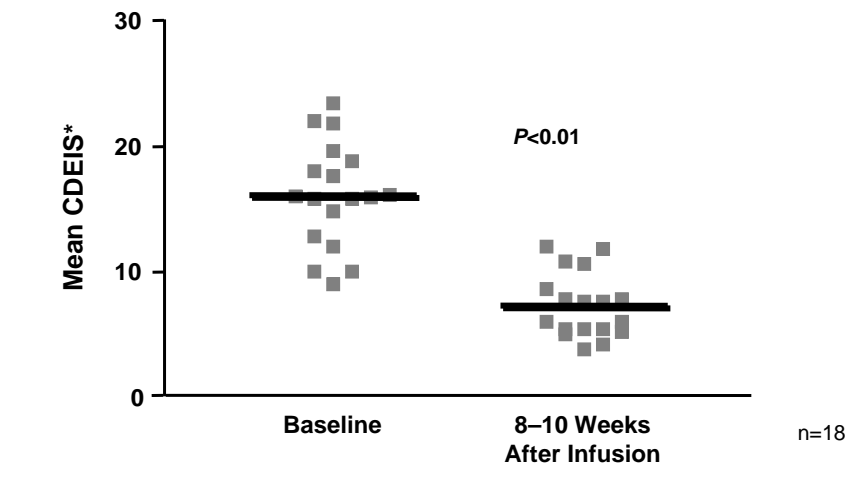

* Crohn's Disease Endoscopic Index of Severity

Mean Value

Fig. (3). Significant endoscopic healing following IFX therapy in paediatric patients with $\mathrm{CD}[63]$.

As of December 2007, there were no published data on the use of adalimumab or certolizumab pegol in paediatric CD.

\section{PRACTICAL ISSUES WHEN TREATING PATIENTS WITH IFX}

\section{Optimising Treatment Regimen}

Traditionally, a 'step-up' (SU) approach to treatment has been adopted that starts with an aminosalicylate and/or antibiotics for mild cases, with corticosteroids for those who fail to respond or who relapse and for moderate to severe cases [64]. Although prescribing of aminosalicylates is widespread, the use of these agents is being questioned. Exclusive nutritional therapy is used as a primary option in some centres for motivated patients. Immunosuppressive agents (AZA, 6-MP, MTX) are most frequently added as secondline treatment, while biologic therapy (e.g., IFX) remains a last resort when all other therapies have failed to induce remission.
The combined corticosteroid/immunomodulator therapy approach often remains unsatisfactory because it has been shown to lead to only a $26 \% 1$-year remission rate, steroid dependency/resistance and toxicity in adults [65]. One paediatric study has evaluated the 3-month and 1-year outcomes (complete response, partial response and corticosteroid resistance) in 109 children with moderate to severe CD who were treated with corticosteroids within 30 days of diagnosis. The study found that at 3 months, most children (84\%) had complete or partial remission (17\% were steroid resistant); at 1 year, despite concomitant use of immunomodulators, $31 \%$ were corticosteroid dependent, and $8 \%$ required surgery [66]. This study also evaluated the effect of IFX on outcomes: IFX improved outcomes in corticosteroid-dependent/-resistant patients and allowed the majority of patients to rapidly discontinue corticosteroid therapy [66]. These data support the current clinical use of SU therapy in paediatrics.

Some pilot studies were undertaken to examine whether a 'top-down' (TD) strategy to treat CD would be more effective in achieving long-term remission. Hommes et al. examined whether earlier induction therapy with IFX and AZA produced higher remission rates (defined as CDAI $<150$ without corticosteroids and no resection) than the traditional 'step-up' strategy [67]. In total, 133 adult CD patients (CDAI >220) diagnosed for less than 4 years and never treated with corticosteroids, immunomodulators or IFX were randomised to receive TD therapy (IFX plus AZA) or SU therapy (aminosalicylate plus topical/systemic corticosteroids as clinically necessary). The study demonstrated that TD therapy leads to higher and more prolonged remission rates without steroids and increased mucosal healing. At 6 months, $60 \%$ of patients receiving TD therapy compared with $41 \%$ in the SU group were in remission. In the SU group, $31 \%$ of patients were still receiving corticosteroid therapy but none were in the TD group. However, fewer patients in the SU group were using immunomodulators compared to the TD group and serious adverse events were high in both groups.

A retrospective paediatric study has compared the effect of SU and TD therapy in 32 children with newly diagnosed CD [68]. The study compared first-line IFX therapy plus AZA or MTX) with traditional (non-biologic) SU therapy (long-term AZA or MTX). Patients who received IFX $(\mathrm{n}=13)$ were treated with $5 \mathrm{mg} / \mathrm{kg}$ at weeks 0,2 and 6 , and 9 patients also received retreatment every 8 weeks for 12 months. In the long-term AZA or MTX group ( $n=19), 5$ patients initially received nutritional course alone and 14 patients initially received corticosteroids. At baseline, after remission and throughout the study, ileocolonoscopy with histology and clinical assessment using the PCDAI were performed. Initial therapy with either IFX or a traditional regimen induced clinical remission [68]. The rate of clinical relapse at 1 year, however, was significantly lower in patients receiving IFX than in those receiving non-biologic therapy (Fig. 4). Furthermore, the CDEIS score was lower in patients receiving IFX (6.5) than in those receiving nonbiologic therapy (12.4). This shows that IFX suppresses intestinal inflammation and promotes a more prolonged and stable remission. These preliminary data suggest that earlier treatment with IFX may have number of potential benefits; however, additional studies are warranted. 


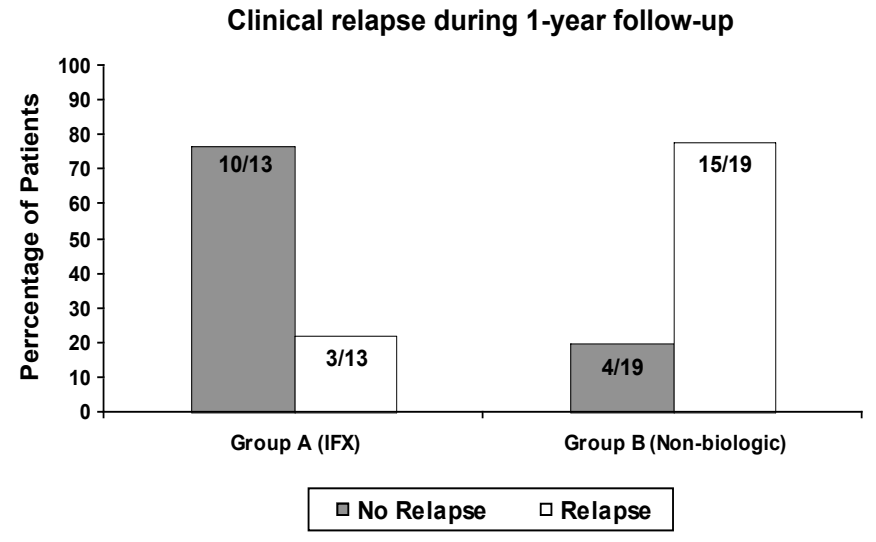

Fig. (4). Early IFX versus traditional (non-biologic) therapy in paediatric CD [68].

\section{Minimising Potential Risks of IFX Therapy}

\section{Immunogenicity}

Treatment with exogenous proteins such as monoclonal antibodies has the potential for the development of antibodies to these proteins [69]. Maintenance therapy studies in adult $\mathrm{CD}$ have demonstrated rates of antibodies to adalimumab and certolizumab pegol to be approximately $2.6 \%$ [70] and $8 \%$ [71], respectively. Long-term studies in adults with $\mathrm{CD}$ have shown that antibodies to IFX develop in about $7 \%$ to $10 \%$ of patients receiving scheduled maintenance infusions (every 4-8 weeks) [57, 72]. In the REACH paediatric CD study, of the patients who were evaluated for the presence of antibodies to IFX through week 54, 2.9\% (3/105) were positive for antibodies [61]. The presence of antibodies to IFX may develop and have been associated with an increased frequency of infusion reactions [38]. In clinical trials, delayed hypersensitivity reactions have been reported and available data suggest an increased risk for delayed hypersensitivity with increasing drug-free interval [38]. It has been shown, however, that the use of scheduled rather than episodic IFX therapy reduces antibody formation [69]. In addition, the development of antibodies to IFX can be reduced further with concomitant immunomodulator use (Fig. 5) [69].

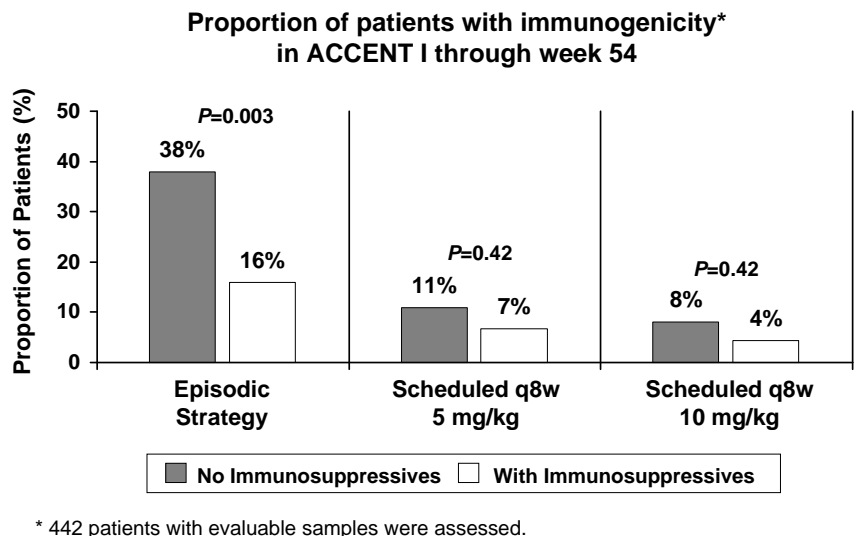

Fig. (5). The effect on immunogenicity of scheduled/episodic IFX therapy in adult CD with concomitant immunomodulator use [69]. Adapted with permission from Elsevier Limited.

\section{Lymphoma}

Immunosuppression has been shown to be linked to an increased risk of lymphoma. A meta-analysis of 6 studies has evaluated the relative risk of lymphoma in patients with IBD treated with AZA or 6-MP. It was found that the risk of lymphoma was 4 times higher in patients treated with AZA/6MP [73]. In the controlled clinical trials of TNF-blocking agents, more cases of malignancies including lymphoma have been observed among patients receiving a TNF blocker compared with control patients $[38,74]$. During clinical trials of adalimumab across all approved indications, the rate of lymphomas was low and similar to the control patients [74]. During clinical trials of IFX across all approved indications, the incidence of lymphoma in IFX-treated patients was higher than expected in the general population, but the occurrence of lymphoma was rare [38]. Cases of hepatosplenic T-cell lymphoma (HSTCL), a rare and very aggressive form of non-Hodgkin's lymphoma, have been associated with IFX therapy. Up to October 2006, 8 of 100 cases of HSTCL reported were associated with IFX therapy [75]; however, the primary role of IFX has not been established. All of the patients who developed HSTCL were also taking chronically concomitant, high doses of immunosuppressants (mainly 6MP or azathioprine) [75, 76]. Indeed, several studies have shown that IFX therapy does not increase the risk of lymphoma. Findings from the TREAT registry in adult patients, which was set up to monitor the long-term safety of IFX and other CD treatments, have shown that there is no significant increase in the incidence of lymphoma with IFX therapy. In total, 6290 patients were assessed (3179 had received IFX). The incidence of lymphoma per 100 patient-years was 0.062 for IFX patients compared with 0.057 for those who had not received IFX [77, 78]. The incidence of all cancer was also similar (Fig. 6). This is supported by the findings of a multicentre, matched-pair study that compared the cancer risk in CD patients treated with IFX with those who were not. The frequency of new neoplasia diagnosis was found to be comparable between the 2 patient groups [79].

\section{Number of malignancies per 100 patient years}

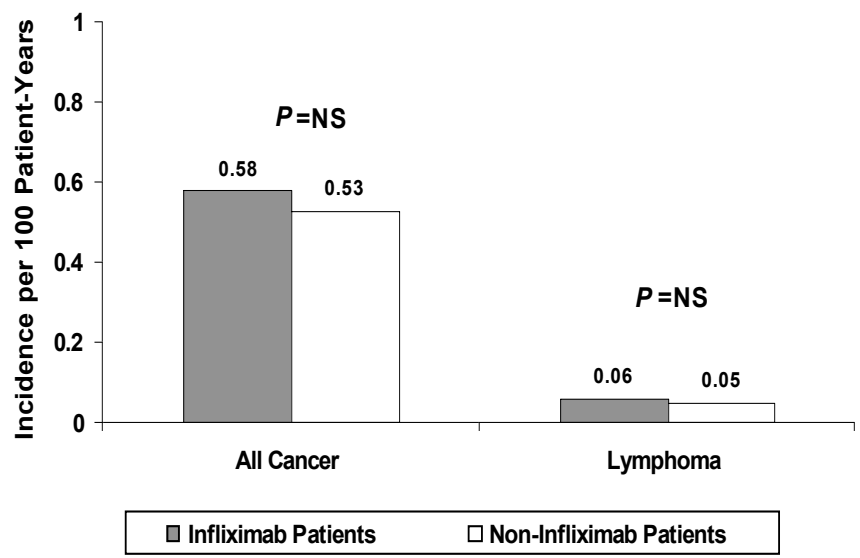

Fig. (6). The incidence of lymphoma with or without IFX treatment [77]. 


\section{Opportunistic Infection}

In the body, TNF $\alpha$ mediates inflammation and modulates immune responses and is essential for the removal of intracellular infections. Antagonism of TNF $\alpha$, therefore, may compromise the defense against infection in some patients [38]. Tuberculosis (TB), bacterial infections, including sepsis and pneumonia, invasive or superficial fungal infections and other opportunistic infections have been observed in patients treated with adalimumab and IFX $[38,74]$. A relationship has been shown between the use of TNF antagonists and the onset of granulomatous infection, most commonly TB [80]. The FDA Adverse Event Reporting System has reported that TB occurred in $144 / 100,000$ patients with IFX and in 35/100,000 patients with etanercept between 1998 through the third quarter of 2002. The clustering of reports shortly after initiation of IFX treatment, however, is consistent with reactivation of latent disease in patients not exhibiting any symptoms rather than the initiation of new disease [80]. The majority of this information is based on adult data; however, children may be at risk for developing infections; therefore, their immunisations should be up to date.

Opportunistic infections reported in patients on IFX have included but are not limited to pneumocystosis, histoplasmosis, cytomegalovirus, atypical mycobacterium, listeriosis and aspergillosis [38]. Some of these opportunistic infections have also been observed with adalimumab [74]. The TREAT registry found an increased incidence of serious infections with IFX, but it was determined that the risk was due to prednisone and disease severity and not IFX [77, 78].

\section{Treatment Considerations}

Patient selection is important when considering treatment with IFX. Certainly not all paediatric patients with $C D$ will require IFX therapy and overtreatment should be avoided. Current guidelines recommend that IFX be used in children with severe, active disease who fail conventional therapy, including a corticosteroid, an immunomodulator and primary nutrition therapy $[38,81]$.

Preliminary data suggest that earlier introduction of biologic therapy may be more effective, especially for achieving mucosal healing which may influence the course of the disease. With regard to costs, a recent review suggests it appears that earlier management with anti-TNF therapies has the potential to lower overall costs for the treatment of $\mathrm{CD}$, despite the higher cost of medication [82]. Because limited data exist in both adult and pediatric populations, more studies are warranted to determine the cost benefit between SU $v s$ TD treatment approaches. Furthermore, it has been shown that children with early, refractory and/or fistulising CD $(<1$ year) are more likely to achieve a prolonged response to IFX than those with late disease ( $>1$ year) [83], suggesting that early use of IFX is beneficial in these patients. These features have also been confirmed in an earlier study by Kugathasan et al. [84]. However, it may not be clear from the first presentation whether the patient has CD and longterm safety remains a concern.

In addition, in adult patients, scheduled rather than episodic dosing is preferable as it not only leads to longer lasting remission and more complete mucosal healing [58], but also reduces the risk of immunogenicity. In paediatric $\mathrm{CD}$,
IFX has only been used in combination with conventional immunosuppressive therapy [38]. Although there is little or no evidence to show that adding an immunomodulator to IFX therapy results in improved outcomes (i.e., remission maintenance/mucosal healing), concomitant therapy does lower the incidence of antibodies to IFX and infusion reactions [38]. Recent observations indicate that long-term continuation of immunosuppressives offers no clinical benefit over IFX monotherapy but is associated with higher IFX trough levels; these features are more evident beyond 6 months of combined IFX and immunomodulator therapy [85].

If acute infusion reactions do occur, infusions should be stopped immediately. Patients may be pretreated with an antihistamine or hydrocortisone to prevent mild and transient effects [38]. Patients must be screened for infections, including TB, prior to initiating IFX treatment and monitored for opportunistic infections during and for 6 months after treatment has finished [38]. Additionally, patients should receive the patient alert card from their physicians and record important information.

\section{CONCLUSIONS}

The incidence of $\mathrm{CD}$ has been increasing among paediatric patients. Although adult and paediatric disease is similar, there is a higher risk of disabling/complicated disease in children. In addition, up to a quarter of children will suffer serious extraintestinal manifestations, particularly growth retardation and delayed sexual development. Consequently, it is essential to diagnose and treat paediatric CD early. The aim of therapy in children should be to not only achieve symptomatic remission, but also to impact disease progression. The recent advent of IFX to treat children who fail nonbiologic therapies has changed the paradigm. IFX has been shown to be highly effective in inducing and maintaining prolonged remission in moderate to severe, active disease. IFX restores growth and improves QoL. Importantly, IFX induces intestinal healing and therefore has the ability to allow the bowel to return to normal function and thus change the course of the disease if used early in paediatric CD. Earlier use of biologic therapies such as IFX may be more beneficial to the patient than traditional strategies. Large paediatric databases, scheduled therapy and prospective, wellcontrolled paediatric trials are ongoing and mandatory to address these crucial issues.
ABBREVIATIONS

$\begin{array}{ll}\text { AZA } & =\text { Azathioprine } \\ \mathrm{CBC} & =\text { Complete blood count } \\ \mathrm{CD} & =\text { Crohn's disease } \\ \mathrm{CDEIS} & =\text { Crohn's Disease Endoscopic Index of Severity } \\ \mathrm{ESR} & =\text { Erythrocyte sedimentation rate } \\ \mathrm{GI} & =\text { Gastrointestinal } \\ \mathrm{HSTCL} & =\text { Hepatosplenic T-cell lymphoma } \\ \mathrm{IBD} & =\text { Inflammatory bowel disease } \\ \mathrm{IFX} & =\text { Infliximab } \\ \text { MTX } & =\text { Methotrexate }\end{array}$




$$
\begin{array}{ll}
\text { QoL } & =\text { Quality of life } \\
\text { PCDAI } & =\text { Pediatric Crohn's Disease Activity Index } \\
\text { RCT } & =\text { Randomised controlled trials } \\
\text { SU } & =\text { Step-up } \\
\text { TB } & =\text { Tuberculosis } \\
\text { TD } & =\text { Top-down } \\
\text { TNF } & =\text { Tumour necrosis factor } \\
\text { TPMT } & =\text { Thiopurine methyltransferase } \\
6-M P & =6-M e r c a p t o p u r i n e
\end{array}
$$

\section{ACKNOWLEDGEMENT}

Editorial support for the development of this publication was provided by Schering-Plough Corporation.

\section{REFERENCES}

[1] Mamula P, Markowitz JE, Baldassano RN. Inflammatory disease in early childhood and adolescence: special considerations. Gastroenterol Clin N Am 2003; 32: 967-95.

[2] Danzi JT. Extraintestinal manifestations of idiopathic inflammatory bowel disease. Arch Intern Med 1998; 148: 297-302.

[3] Hyams JS. Crohn's disease in children. Pediatr Clin N Am 1996; 43: 255-77.

[4] Burbige EJ, Huang SH, Bayliss TM. Clinical manifestations of Crohn's disease in children and adolescents. Pediatrics 1975; 55: 866-71.

[5] Lindsley CB, Schaller JG. Arthritis associated with inflammatory bowel disease in children. J Pediatr 1974; 84: 16-20.

[6] Baldassano RN, Piccoli DA. Inflammatory bowel disease in pediatric and adolescent patients. Gastroenterol Clin N Am 1999; 28: 445-58.

[7] Booth IW. The nutritional consequences of gastrointestinal disease in adolescence. Acta Paediatr Scand Suppl 1991; 373: 91-102.

[8] Brain CE, Savage MO. Growth and puberty in chronic inflammatory disease. Bailliéres Clin Gastroenterol 1994; 8: 83-100.

[9] Seidman E, LeLeiko N, Ament M, et al. Nutritional issues in pediatric inflammatory bowel disease. J Pediatr Gastroenterol 1991; 12: 424-38.

[10] Kanof ME, Lake AM, Bayless TM. Decrease height velocity in children and adolescents before diagnosis of Crohn's disease. Gastroenterology 1988; 95: 1523-7.

[11] Logan RF. Inflammatory bowel disease incidence: up, down or unchanged? Gut 1998; 42: 309-11.

[12] Russel MG, Stockbrugger RW. Epidemiology of inflammatory disease: an update. Scand Gastroenterol 1996; 31: 417-27.

[13] Oliva-Hemka M, Fiocchi C. Etiopathogenesis of inflammatory bowel disease: the importance of the pediatric perspective. Inflamm Bowel Dis 2002; 8: 112-28.

[14] Cosgrove M, Al-Atia RF, Jenkins HR. The epidemiology of pediatric inflammatory disease. Arch Dis Child 1996; 74: 460-1.

[15] Kim S, Ferry G. Inflammatory bowel disease in children. Curr Probl Pediatr Adolesc Health Care 2002; 32: 108-32.

[16] Seidman EG. Recent advances in the diagnosis and treatment of pediatric inflammatory disease. Curr Gastroenterol Rep 2000; 2: 248-52.

[17] Sawczenko A, Sandhu BK, Logan RFA, et al. Prospective survey of childhood inflammatory bowel disease in the British Isles. Lancet 2001; 357: 1093-4

[18] Griffiths AM, Hugot JP. In: Walker AW, Goulet O, Kleinman R, Sherman P, Shneider B, Sanderson I, eds. Pediatric Gastrointestinal Disease: Pathophysiology, Diagnosis, Management. 4th ed. Philadelphia, PA: BC Decker Inc 2004; pp. 789-824.

[19] Engström I. Inflammatory bowel disease in children and adolescents: mental health and family functioning. J Pediatr Gastroenterol Nutr 1999; 28: S28-33.

[20] Carvalho R, Hyams JS. Diagnosis and management of inflammatory bowel disease in children. Semin Pediatr Surg 2007; 16: 16471 .
[29] Lenaerts C, Roy CC, Vaillancourt M, Weber AM, Morin CL, Seidman E. High incidence of upper gastrointestinal tract involvement in children with Crohn's disease. Pediatrics 1989; 83: 777-81.

[30] Cameron DJ. Upper and lower gastrointestinal endoscopy in children and adolescents with Crohn's disease: a prospective study. J Gastroenterol Hepatol 1991; 6: 355-58.

[31] Coremans G, Ectors N, Hoffman I, Veereman-Wauters G. Is childhood Crohn's disease a reasonable indication for esophago-gastroduodenoscopy? Gastroenterology 1998; 114(Suppl 4): A955.

[32] Farmer RG, Michener WM. Prognosis of Crohn's disease with onset in childhood or adolescence. Dig Dis Sci 1979; 24: 752-7.

[33] Markowitz J, Daum F, Agis H, Kahn E, Silverberg M, Fisher SE. Perianal disease in children and adolescents with Crohn's disease. Gastroenterology 1984; 86: 829-33.

[34] Mamula P, Telega GW, Markowitz JE, et al. Inflammatory bowel disease in children 5 years of age and younger. Am J Gastroenterol 2002; 97: 2005-10.

[35] Escher J. Diagnostic work-up and disease phenotype in pediatric IBD: 2 year results from the ESPGHAN registry. J Pediatr Gastroenterol Nutr 2006; 43(Suppl 2): S8-10.

[36] Ruemmele FM, El Khoury MG, Talbotec C, et al. Characteristics of inflammatory bowel disease with onset during the first year of life. J Pediatr Gastroenterol Nutr 2006; 43: 603-9.

[37] Markowitz J, Grancher K, Kohn N, Lesser M, Daum F. A multicenter trial of 6-mercptopurine and prednisone in children with newly diagnosed Crohn's disease. Gastroenterology 2000; 119: 895-902.

[38] Remicade ${ }^{\circledR}$ (infliximab) Summary of Product Characteristics (SPC). October 2007.

[39] Akobeng K, Gardener E. Oral 5-aminosalicylic acid for maintenance of medically-induced remission in Crohn's disease. Cochrane Database Syst Rev 2005; (1): CD003715.

[40] Steinhart AH, Ewe K, Griffiths AM, Modigliani R, Thomsen OO. Corticosteroids for maintenance of remission in Crohn's disease. Cochrane Database Syst Rev 2003; (4): CD000301.

[41] Keenan GF. Management of complications of glucocorticoid therapy. Clin Chest Med 1997; 16: 1-17.

[42] Mrakotsky C, Bousvaros A, Chriki L. Impact of acute steroid treatment on memory, executive function, and mood in pediatric inflammatory bowel disease. J Pediatr Gastroenterol Nutr 2005; 41: 540-1.

[43] Tung J, Loftus EV, Freese DK, et al. A population-based study of the frequency of corticosteroid resistance and dependence in pediatric patients with Crohn's disease and ulcerative colitis. Inflamm Bowel Dis 2006; 12: 1093-100.

[44] Kirschner BS, Klich JR, Kalman SS, deFavaro MV, Rosenberg IH. Reversal of growth retardation in Crohn's disease with therapy emphasizing oral nutritional restitution. Gastroenterology 1981; 80: 10-5.

[45] Papadopoulou A, Holden CE, Paul L, Sexton E, Booth IW. The nutritional response to home enteral nutrition in childhood. Acta Paediatr 1995; 84: 528-31. 
[46] Griffiths AM, Ohlsson A, Sherman PM, Sutherland LR. Metaanalysis of enteral nutrition as a primary treatment of active Crohn's disease. Gastroenterology 1995; 108: 1056-67.

[47] Borrelli O, Cordischi L, Cirulli M, et al. Polymeric diet alone versus corticosteroids in the treatment of active pediatric Crohn's disease: a randomized controlled open-label trial. Clin Gastroenterol Hepatol 2006; 4: 744-53.

[48] Baldassano RN, Han PD, Jeshion WC, et al. Pediatric Crohn's disease: risk factors for postoperative recurrence. Am J Gastroenterol 2001; 96: 2169-76.

[49] McLain BI, Davidson PM, Stokes KB, Beasley SW. Growth after gut resection for Crohn's disease. Arch Dis Child 1990; 65: 760-2.

[50] Griffiths AM, Wesson DE, Shandling B, Corey M, Sherman PM. Factors influencing postoperative recurrence of Crohn's disease in children. Gut 1991; 32: 491-5.

[51] Gryboski JD. Crohn's disease in children 10 years old and younger: comparison with ulcerative colitis. J Pediatr Gastroenterol Nutr 1994; 18: 174-82.

[52] Griffiths AM, Nguyen P, Smith C, MacMillan JH, Sherman PM. Growth and clinical course of children with Crohn's disease. Gut 1993; 34: 939-43

[53] Branch J, Baldé M, Vernier-Massouille G, et al. Risk factors for initial surgery in pediatric patients with Crohn's disease: a population-based cohort study. Gut 2007; 56(Suppl 3): OP-G-341.

[54] Homer DR, Grand RJ, Colodny AH. Growth, course, and prognosis after surgery for Crohn's disease in children and adolescents. Pediatrics 1977; 59: 491-5.

[55] Scallon B, Cai A, Solowski N, et al. Binding and functional comparisons of two types of tumor necrosis factor antagonists. J Pharmacol Exp Ther 2002; 301: 418-26.

[56] Targan SR, Hanauer SB, Sander JH, et al. A short-term study of chimeric monoclonal antibody cA2 to tumor necrosis factor $\alpha$ for Crohn's disease. N Engl J Med 1997; 337: 1029-35.

[57] Hanauer SB, Feagan BG, Lichtenstein GR, et al. Maintenance infliximab for Crohn's disease: the ACCENT I randomised trial. Lancet 2002; 359: 1541-9.

[58] Rutgeerts P, Feagan BG, Lichtenstein GR, et al. Comparison of scheduled and episodic treatment strategies of infliximab in Crohn's disease. Gastroenterology 2004; 126: 402-13.

[59] Lichtenstein GR, Yan S, Bala M, Blank M, Sands BE. Infliximab maintenance treatment reduces hospitalizations, surgeries, and procedures in fistulizing Crohn's disease. Gastroenterology 2005; 128 : 862-9.

[60] Remicade ${ }^{\circledR}$ (infliximab) [prescribing Information]. Malvern, PA: Centocor, Inc.; 2007.

[61] Hyams J, Crandall W, Kugathasan S, et al; REACH Study Group. Induction and maintenance infliximab therapy for the treatment of moderate-to-severe Crohn's disease in children. Gastroenterology 2007; 132: 863-73.

[62] Walters TD, Gilman AR, Griffiths AM. Linear growth improves during infliximab therapy in children with chronically active severe Crohn's disease. Inflamm Bowel Dis 2007; 13: 424-30.

[63] Borrelli O, Bascietto C, Viola F, et al. Infliximab heals intestinal inflammatory lesions and restores growth in children with Crohn's disease. Dig Liver Dis 2004; 36: 342-7.

[64] Hanauer SB, Sandborn W. Practice Parameters Committee of the American College of Gastroenterology. Management of Crohn's disease in adults. Am J Gastroenterol 2001; 96: 635-43.

[65] Stokkers PC, Hommes DW. Novel biological therapies for IBD. Curr Treat Options Gastroenterol 2006; 9: 201-10.

[66] Markowitz J, Hyams J, Mack D, et al; Pediatric IBD Collaborative Research Group. Corticosteroid therapy in the age of infliximab: acute and 1-year outcomes in newly diagnosed children with Crohn's disease. Clin Gastroenterol Hepatol 2006; 4: 1124-9.
[67] Hommes D, Beart F, van Assche G, et al. The ideal management of Crohn's disease: top down versus step up strategies, a randomised controlled trial. Gastroenterology 2006; 130(Suppl 2): A-749.

[68] Romeo E, Viola F, De Angelis G, et al. Infliximab as a first choice therapy in children with newly diagnosed Crohn's disease (CD) promotes long-term sustained remission and alters the course of the disease. Gastroenterology 2006; 130(Suppl 2): A56.

[69] Hanauer SB, Wagner CL, Bala M, et al. Incidence and importance of antibody responses to infliximab after maintenance or episodic treatment in Crohn's disease. Clin Gastroenterol Hepatol 2004; 2: 542-53.

[70] Sandborn WJ, Hanauer SB, Rutgeerts P, et al. Adalimumab for maintenance treatment of Crohn's disease: results of the CLASSIC II trial. Gut 2007; 56: 1232-9.

[71] Schreiber S, Khaliq-Kareemi M, Lawrance IC, et al; PRECISE 2 Study Investigators. Maintenance therapy with certolizumab pegol for Crohn's disease. N Engl J Med 2007; 357: 239-50.

[72] Maini RN, Breedveld FC, Kalden JR, et al; Anti-Tumor Necrosis Factor Trial in Rheumatoid Arthritis with Concomitant Therapy Study Group. Sustained improvement over two years in physical function, structural damage and signs and symptoms among patients with rheumatoid arthritis treated with infliximab and methotrexate. Arthritis Rheum 2004; 50: 1051-65.

[73] Kandiel A, Fraser AG, Korelitz BI, Brensinger C, Lewis JD. Increased risk of lymphoma among inflammatory bowel disease patients treated with azathioprine and 6-mercaptopurine. Gut 2005; 54: $1121-5$.

[74] Humira ${ }^{\circledR}$ (adalimumab) Summary of Product Characteristics (SPC). October 2007.

[75] Mackey AC, Green L, Liang L, Dinndorf P, Avigan M. Hepatosplenic $\mathrm{T}$ cell lymphoma associated with infliximab use in young patients treated for inflammatory bowel disease. J Pediatr Gastroenterol Nutr 2007; 44: 265-7.

[76] Data on File. Centocor, Inc.

[77] Lichtenstein GR, Cohen RD, Feagan BG, et al. Safety of infliximab and other Crohn's disease therapies-updated TREATTM Registry data over 10,000 patient years of follow up. Gastroenterology 2006; 130(Suppl 2): A71.

[78] Lichtenstein GR, Feagan BG, Cohen RD, et al. Serious infections and mortality in association with therapies for Crohn's disease: TREAT Registry. Clin Gastroenterol Hepatol 2006; 4: 621-30.

[79] Biancone L, Orlando A, Kohn A, et al. Infliximab and newly diagnosed neoplasia in Crohn's disease: a multicentre match pair study. Gut 2006; 55: 228-33.

[80] Wallis RS, Broder MS, Wong JY, Hanson ME, Beenhouwer DO Granulomatous infectious disease associated with tumor necrosis factor antagonists. Clin Infect Dis 2004; 38: 1261-5.

[81] Working Group of the Japanese Society for Pediatric Gastroenterology, Hepatology and Nutrition, Konno M, Kobayashi A, et al. Guidelines for the treatment of Crohn's disease in children. Pediatr Int 2006; 48: 349-52.

[82] Hanauer SB. Turning traditional treatment strategies on their heads: current evidence for "step-up" versus "top-down". Rev Gastroenterol Disord 2007; 7(Suppl 2): S17-S22.

[83] Lionetti P, Bronzini F, Salvestrini C, et al. Response to infliximab is related to disease duration in pediatric Crohn's disease. Aliment Pharmacol Ther 2003; 18: 425-31.

[84] Kugathasan S, Werlin SL, Martinez A, et al. Prolonged duration of response to infliximab in early but not late pediatric Crohn's disease. Am J Gastroenterol 2000; 95: 3189-94.

[85] Van Assche G, Paintaud G, Magdelaine C, et al. Concomitant immunosuppression does not impact the outcome of maintenance infliximab therapy in Crohn's disease: final results of the IMID trial. Gastroenterology 2007; 132(Suppl 2): A-734. 